\title{
5-HYDROXYTRYPTAMINE: A SUBSTRATE FOR RAT LIVER MITOCHONDRIAL MONOAMINE OXIDASE-A AND -B*
}

\author{
Hiroyasu KINEMUCHI, Takamori KAWAGUCHI, Yuichiro ARAI, \\ Hirohisa TAJIMA, Lars ORELAND** and Kazuya KAMIJO \\ Department of Pharmacology, School of Medicine, Showa University. \\ 1-5-8 Hatanodai. Shinagawa-ku, Tokyo 142. Japan
}

Accepted June 17, 1982

On the basis of substrate specificities and inhibitor sensitivities, mitochondrial monoamine oxidase (MAO) (EC 1.4.3.4) has been classified as two forms, MAO-A and MAO-B (1). In rat liver, serotonin is preferentially oxidized by MAO-A, which is highly sensitive to inhibition by clorgyline but less so to deprenyl $(1,2)$. Benzylamine and $\beta$-phenylethylamine (PEA) are preferentially oxidized by MAO-B, and tyramine is oxidized by both forms of MAO (1. 2). However, these specificities are not always observed ( 3 ) and it has also been found that MAO-B in some tissues can oxidize serotonin to some extent (4-8). Our previous studios on rat brain MAO revealed that PEA, long regarded as a specific substrate for MAO-B, is also oxidized by MAO-A and that the ratio of MAO-A to MAO-B activity with PEA is concentration-dependent (9-13).

Here, we investigated the concentrationdependence of the oxidation rates of serotonin by the two forms of MAO in rat liver mitochondria. We also oxamined whether the oxidation with MAO-A of rat liver has a "ping-pong" or double-displacement reaction mechanism like that shown for MAO-B in a variety of tissues (14-18).

Rat liver mitochondria were prepared by differential centrifugation of a homogenate in

*Tlis work was supported in part by a grant from the Ministry of Education, Science and Culture. Japan.

**Present address: Department of Pharmacology. Umea Univorsity, S-901 87. Umea, Sweden.
$0.25 \mathrm{M}$ sucrose-0.01 M phosphate buffer, $\mathrm{pH}$ 7.4. MAO activity was determined radiochemically at $37^{\circ} \mathrm{C}$ and $\mathrm{pH} 7.4$ as described previously (18). Unless otherwise specified. in inhibition studies. mitochondria were preincubated for $30 \mathrm{~min}$ with either clorgyline or deprenyl at $37^{\circ} \mathrm{C}$ before adding substrate. The remaining activity was then determined radiochemically (18). In experiments in which the concentration of oxygen $(54.3-542.5 / / \mathrm{M})$ was changed, the reaction mixture was bubbled for 5 sec with different mixtures of oxygen and nitrogen.

The protein concentration was determined by the modified biuret method (19) with bovine serum albumin as standard.

The inhibitions by clorgyline and deprenyl of MAO activity towards different concentrations of serotonin were studied and results with clorgyline are shown in Fig. 1. The curves for inhibition by both inhibitors differed with the concentration of serotonin. In accordance with the concept that serotonin is a preferential substrate of MAO-A $(1,2)$, at low concentrations $(0.01-0.05 \mathrm{mM})$, singlesigmoidal inhibition curves with a higher sensitivity to clorgyline than deprenyl were obtained. In contrast, at higher concentrations of serotonin $(0.5$ and $5 \mathrm{mM})$, inhibition curves gradually became doublesigmoidal (Fig. 1). These results are anologous to those reported for PEA, which was found to be oxidized differentially by the two forms of MAO in a concentration- 


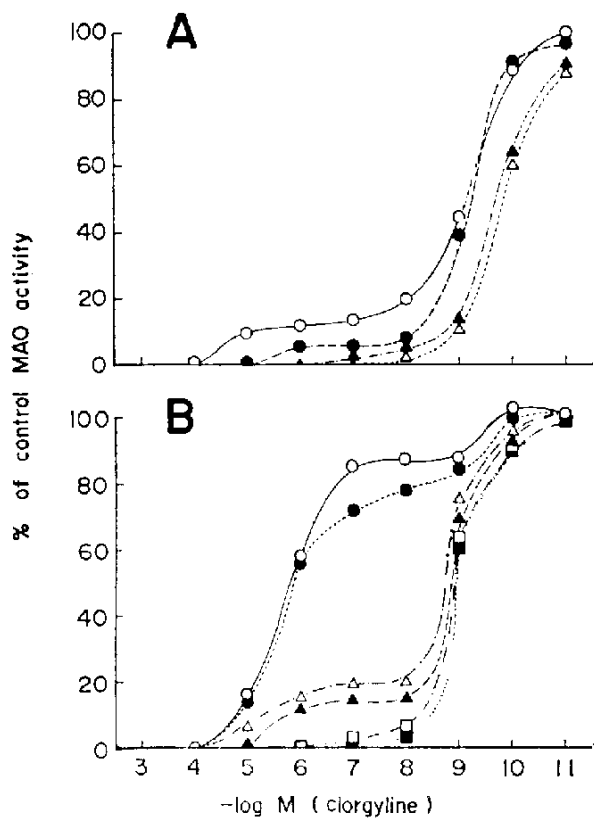

Fig. 1. Effect of clorgyline on MAO activity in untreated, clorgyline-treated and deprenyltreated rat liver mitochondria. For $A$, mitochondria ( $1 \mathrm{mg} / \mathrm{ml}$ protein) were preincubated at $37^{\circ} \mathrm{C}$ for $30 \mathrm{~min}$ with clorgyline before estimation of activity. For $B$, mitochondria were first preincubated with either $2 \mathrm{nM}(\triangle . \Delta)$ or $3.3 \mathrm{nM}(\mathrm{O}, \mathbf{0})$ clorgyline or $3.3 \mathrm{nM}$ ( 1. deprenyl for $60 \mathrm{~min}$, and then samples were further preincubated with different concentrations of clorgyline for an additional $30 \mathrm{~min}$. (A): MAO activity in untreated mitochondria was estimated with $0.01(\Delta), 0.05(\boldsymbol{\Delta}), 0.5(\mathbf{O})$, or $5 \mathrm{mM}(\mathrm{O})$ serotonin as substrate and expressed as a percentage of the control activity in the absence of clorgyline. (B); MAO activity was estimated with $0.5(O . \triangle . \square)$, or $5 \mathrm{mM}$

D) serotonin as substrate and expressed as a percentage of the activity in preparations preincubated for $90 \mathrm{~min}$ in the presence of 2 or $3.3 \mathrm{nM}$ clorgyline or $3.3 \mathrm{nM}$ deprenyl. Each point represents the mean of triplicate determinations of activity in three groups of mitochondrial preparations, each derived from the livers of four rats.

dependent manner (9-13, 20).

None of the clorgyline-resistant activity towards serotonin at high concentrations was due to soluble amine oxidase, which is reversibly inhibited by clorgyline (21) or to amine oxidase, which is insensitive to clorgyline and deprenyl (22), since KCN and semicarbazide ( $1 \mathrm{mM}$ ), inhibitors of these two enzymes, did not change the doublesigmoidal inhibition curves of activity and the activity could be inhibited completely by either clorgyline or deprenyl.

In separate experiments in which mitochandria were preincubated with 3.3 or $2 \mathrm{nM}$ clorgyline, concentration-dependent inhibitions of $50-90 \%$ of the serotonin oxidation were observed. When $0.01 \mathrm{mM}$ serotonin was used and the remaining activity was then inhibited by clorgyline, the curve was also single-sigmoidal, and shifted to lower concentrations of clorgyline (data not shown). since, in the clorgyline-pretreated preparation, less of the active enzyme remained and so it could be titrated with less clorgyline (23). Thus, at this concentration of serotonin, all oxidation was catalyzed by MAO-A, even though MAO-A had been partially and selectively inhibited. When 0.5 and $5 \mathrm{mM}$ serotonin was used, double-sigmoidal curves were obtained with a higher proportion of the less clorgyline sensitive enzyme (MAO-B) activity (Fig. 1B).

In similar experiments with a preparation pretreated with $3.3 \mathrm{nM}$ deprenyl and with 0.5 and $5 \mathrm{mM}$ serotonin as substrate, all the inhibition curves with additional clorgyline were single-sigmoidal, with no appreciable MAO-B activity, and showed higher sensitivity to clorgyline (Fig. 1B). These results reinforced evidence that, in rat liver mitochondria, serotonin at the high concentrations is oxidized by both forms of MAO but with a high proportion of MAO-A activity, since after partial inhibition of MAO-A activity by clorgyline, the shape of the inhibition curves changed considerably and a trace of the MAO-B component was apparent with these concentrations of serotonin.

Lineweaver-Burk plots showed that $\mathrm{Km}$ 
values for serotonin were $60 \mu \mathrm{M}, 40 \mu \mathrm{M}$ and 2 $\mathrm{mM}$ in untreated, deprenyl-treated (MAO-A) and clorgyline-treated mitochondria (MAO-B), respectively, in agreement with the values reported previously (5). The plots with serotonin as substrate for MAO-B in the clorgyline-treated preparation were found to be linear, but the plots for the deprenyltreated preparation (MAO-A) and for the untreated preparation were not linear, deviating with substrate inhibition. These results with serotonin as substrate are similar to those for rat brain MAO with PEA $(10,11,13)$.

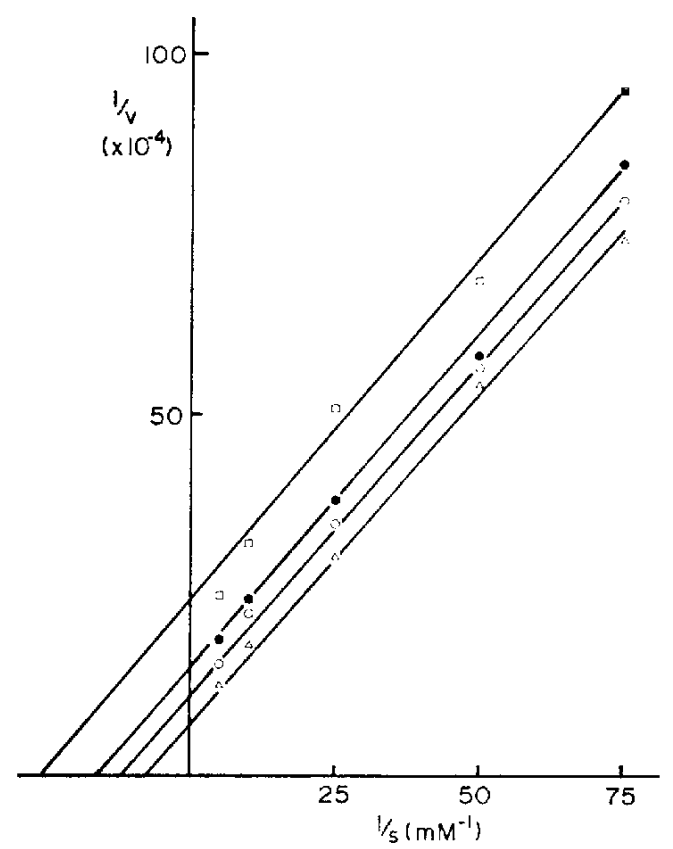

Fig. 2. Lineweaver-Burk plots of MAO-A activity in rat liver mitochondria assayed at different concentrations of oxygen. Ordinate, 1/activity, expressed as cpm $\times 10^{-4}$; abscissa. $1 / \mathrm{mM}$ serotonin. Before assay, mitochondria were preincubated with $33 \mathrm{nM}$ deprenyl for $4 \mathrm{hr}$, and then MAO activities towards different concentrations of serotonin were assaycd under atmospheres of $54.3(-\square-) .108 .5$ (-O-). $217.0(-\mathrm{O}-)$, and $542.5(-\triangle-) \mu \mathrm{M}$ oxygen. All points shown are the means of triplicate determinations of activity in three groups of mitochondrial fractions, each derived from the livers of four rats.
MAO in a variety of tissues has been shown to follow a "ping-pong" or doubledisplacement reaction mechanism (14-18). However, to our knowledge, this mechanism has been demonstrated only for MAO-B, while the mechanism for MAO-A is still unknown. When the MAO-A activity in mitochondria pretreated with deprenyl (33 nM $4 \mathrm{hr}$ ). which completely and irreversibly inhibited MAO-B activity towards low concentrations of PEA (10-12), was assayed using low concentrations (0.013-0.2 mM) of serotonin with decreasing concentrations (542.5-54.3 $\mu \mathrm{M})$, of oxygen results showed uncompetitive inhibition giving parallel plots with a $\mathrm{Km}$ value of $144.9 \mathrm{\mu M}$ for oxygen (Fig. 2). Similar parallel lines were obtained when the oxygen concentration was varied at a series of fixed serotonin concentrations, indicating that $\mathrm{MAO}-\mathrm{A}$ reacts according to the double-displacement reaction mechanism previously shown for MAO-B.

The preferential oxidation of low concentrations of serotonin by MAO-A is presumably due to its lower $\mathrm{Km}$ value $(40 \mu \mathrm{M})$, than that of MAO-B (2 mM). With increasing concentrations of serotonin, the proportion of $\mathrm{MAO}-\mathrm{B}$ activity will increase, since oxidation by MAO-A, which is already at the $V \max$ and which becomes subject to substrate inhibition, cannot increase. The data presented here and previously $(9-13,20)$ show that the concentrations of substrates must be taken into account when the substrate specificities and inhibitor sensitivities of MAO to MAO inhibitors are investigated.

\section{REFERENCES}

1) Neff, N.H. and Yang, H.-Y.T.: Another look at the monoamine oxidases and the monoamine oxidase inhibitor drugs. Life Sci. 14, $2061-$ 2074 (1974)

2) Houslay, M.D., Tipton, K.F. and Youdim, M.B.H.: Muitiple forms of monoamine oxidase: Fact and artefact ? Life Sci. 19, 467-478 (1976)

3) Fowler, C.J., Callingham, B.A., Mantle, T.J. and Tipton, K.F.: Monoamine oxidase A and B: 
A useful concept? Biochem. Pharmacol. 27, 97-101 (1978)

4) Ekstedt, B. and Oreland, L.: Heterogeneity of pig liver and pig brain mitochondrial monoamine oxidase. Archs int. Pharmacodyn. Thér. 222, 157-165 (1976)

5) Ekstedt, B.: Substrate specificity of the different forms of monoamine oxidase in rat liver mitochondria. Biochem. Pharmacol. 25, 1133$1138(1976)$

6) Murphy, D.L., Redmond, D.E., Garrick, N. and Baulu, J.: Brain region differences and some characteristics of monoamine oxidase type A and $B$ activities in the vervet monkey. Neurochem. Fies. 4, 53-62 (1979)

7) Mitra, C. and Guha, S.R.: Serotonin oxidation by type B MAO of rat brain. Biochem. Pharmacol. 29, 1213-1216 (1980)

8) Ekstedt, B.: Substrate specificity of monoamine oxidase in pig liver mitochondria. Med. Biol. 57, 220-223 (1979)

9) Toyoshima, Y., Kinemuchi, $H$. and Kamijo, K.: Nonexistence of a type $\mathrm{C}$ monoamine oxidase in rat brain. J. Neurochem. 32, 1183-1189 (1979)

10) Kinemuchi, H., Wakui, Y., Toyoshima, Y., Hayashi, N. and Kamijo, K.: $\beta$-Phenylethylamine (PEA), a concentration-dependent preferential substrate for multiple forms of MAO. In Monoamine Oxidase: Structure, Function and Altered Function. Edited by, Singer, T.P., Von Korff, R.W. and Murphy, D.L., P. 205-212, Academic Press. Now York (1979)

11) Kinemuchi, $H_{.}$, Wakui, $Y$. and Kamijo, K.: Substrate selectivity of type $A$ and typo $B$ monoamine oxidase in rat brain. J. Neurochem. $35,108-114(1980)$

12) Kinemuchi, H., Wakui, Y., Arai, Y. and Kamijo, K.: Inhibition of monoamine oxidase by clorgyline and deprenyl in circumventricular structure of rat brain. Biochem. Pharmacol. 29 . 2871-2874 (1980)

13) Kinemuchi, H., Arai, Y., Oreland, L., Kamijo, K., Fowler, C.J. and Tipton, K.F.: Inhibition of type B monoamine oxidase activity by $\beta$-phenylethylamine. In Monoamine Oxidase: Basic and Clinical Frontiers, Edited by. Kamijo. K., Usdin,
E. and Nagatsu, T., p. 159-170. Excerpta Medica, Amsterdam (1982)

14) Tipton, K.F.: The reaction pathway of pig brain mitochondrial monoamine oxidase. Europ. J. Biochem. 5, 316-320 (1968)

15) Fischer, A.G., Schulz, A.R. and Oliner, L.: Thyroidal biosynthesis of iodothyronines. II general characteristics and purification of mitochondrial monoamine oxidase. Biochim. Biophys. Acta 159, 460-471 (1968)

16) Oi, S., Shimada, K., Inamasu, M. and Yasunobu, K.T.: Mecharristic studies of beef liver mitochondrial amine oxidase XVIII. Amine oxidase. Archs Biochem. Biophys. 139, 28-37 (1970)

17) Houslay, M.D. and Tipton, K.F.: The reaction pathway of membrane-bound rat liver mitochondrial monoamine oxidase. Biochem. J. 135, 735-750 (1973)

18) Fowler, C.J., Ekstedt, B., Egashira, T., Kinemuchi, H. and Oreland, L.: The interaction between human platelet monoamine oxidase. its monoamine oxidase substrates and oxygen. Bjochem. Pharmacol. 28, 3063-3068 (1979)

19) Yonetani, T.: Studies on cytochrome oxidase. III. Improved preparation and some properties. J. biol. Chem. 236, 1680-1688 (1961)

20) Peers, E.M., Lyles, G.A. and Callingham, B.A.: The deamination of isoamylamine by monoamine oxidase in mitochondrial preparations from rat liver and heart: A comparison with phenylethylamine. Biochem. Pharmacol. 29, 1097-1107 (1980)

21) Houslay, M.D. and Tipton, K.F.: Inhibition of beef plasma amine oxidase by clorgyline. Biochem. Pharmacol. 24, 429-431 (1975)

22) Kinemuchi, H., Morikawa, F., Tajima, H. and Kamijo, K.: Enzymic characteristics of a clorgyline- and deprenyl-resistant amine oxidase in human placenta. in Monoamine Oxidase: Basic and Clinical Frontiers. Edited by, Kamijo. K., Usdin, E. and Nagatsu, T., p. 112-124. Excerpta Medica, Amsterdam (1982)

23) Fowler, C.J., Oreland, L. and Callingham, B.A.: The acetylenic monoamine oxidase inhibitors clorgyline, deprenyl, pargyline and J-508: their properties and applications. J. Pharm. Pharmacol. 33, 341--347 (1981) 